\title{
Morbus Meulengracht
}

Zeitz, Jonas ; Schmidinger, Irène ; Rentsch, Katharina ; Rogler, Gerhard ; Vavricka, Stephan R

DOI: https://doi.org/10.1024/1661-8157/a001212

Other titles: Meulengracht disease

Posted at the Zurich Open Repository and Archive, University of Zurich ZORA URL: https://doi.org/10.5167/uzh-91507

Journal Article

Accepted Version

Originally published at:

Zeitz, Jonas; Schmidinger, Irène; Rentsch, Katharina; Rogler, Gerhard; Vavricka, Stephan R (2013). Morbus Meulengracht. Praxis, 102(3):129-135.

DOI: https://doi.org/10.1024/1661-8157/a001212 


\section{Retrospektive Studie zur Untersuchung der klinischen Symptome und Laborveränderungen bei Patienten mit Meulengracht-Syndrom}

Jonas Zeitz ${ }^{*}$, Iréne Schmidinger ${ }^{2 *}$, Katharina Rentsch ${ }^{3}$, Gerhard Rogler ${ }^{4}$, Stephan R. Vavricka ${ }^{4,5}$

${ }^{1}$ Klinik und Poliklinik für Innere Medizin, UniversitätsSpital Zürich, Rämistrasse 100, 8091 Zürich

2 Universität Zürich, Rämistrasse 71, 8006 Zürich

${ }^{3}$ Insitut für Klinische Chemie, Universitätsspital Basel, Petersgraben 4, 4031 Basel

${ }^{4}$ Klinik für Gastroenterologie und Hepatologie, UniversitätsSpital Zürich, Rämistrasse 100, 8091 Zürich

${ }^{5}$ Klinik für Gastroenterologie und Hepatologie, Stadtspital Triemli, Birmensdorferstrasse 497, 8063 Zürich

${ }^{*}$ contributed equally

\section{Hintergrund und Definition:}

Bilirubin ist ein toxisches Stoffwechselprodukt des Häm-Metabolismus welches in grossen Mengen durch den normalen Umsatz von Hämoglobin und anderen Hämoproteinen ensteht. Täglich werden 250 bis 300mg Bilirubin produziert, $70-80 \%$ entstehen aus dem Abbau alternder Erythrozyten[1,2].

Bilirubin ist wasserunlöslich und daher im Serum an Albumin gebunden. Im endoplasmatischen Retikulum der Hepatozyten wird Bilirubin durch Glucuronidierung metabolisiert. Das Enzym Glucuronyltransferase (UGT) überträgt ein Molekül Glucuronsäure von UDP-Glucuronsäure auf das Substrat. Durch diese Kopplung wird Bilirubin wasserlöslich und kann in die Gallenkanälchen sezerniert und so ausgeschieden werden.

Kommt es zu einem Ungleichgewicht zwischen Produktion und Ausscheidung von Bilirubin führt dies zu einer Serumbilirubinerhöhung. Bilirubin im Serum ist erhöht wenn die Häm-Produktion die metabolische Kapazität von $4 \mathrm{mg} / \mathrm{kg} /$ die oder die Ausscheidung übersteigt[2]. Dies geschieht entweder durch eine zu hohe Abgabe von Bilirubin-Vorstufen in die Blutbahn oder durch eine eingeschränkte hepatische Aufnahme oder Exkretion.

Bemerkbar macht sich dies durch eine Erhöhung des direkten oder indirekten Bilirubins. Eine direkte Hyperbilirubinämie kann beispielsweise durch eine Cholestase, eine Hepatitis oder eine Leberzirrhose verursacht werden.

Bei der indirekten Hyperbilirubinämie kann eine Hämolyse oder eine hereditäre Ursache zugrunde liegen. Dies ist der Fall beim Morbus Meulengracht (oder GilbertSyndrom), dabei handelt es sich um eine milde unkonjugierte Hyperbilirubinämie. Dieses Syndrom wurde erstmals 1906 durch Gilbert et al. beschrieben[3]. Es äussert 
sich durch leicht erhöhte Bilirubinwerte im Blut ohne Bilirubinurie oder andere Anzeichen einer Hämolyse oder Lebererkrankung. Die Erkrankung wird verursacht durch eine verminderte Aktivität des Enzyms UGT1A1, welches hauptsächlich in der Leber exprimiert wird und das nebst endogenen Substanzen wie Bilirubin auch Medikamente metabolisiert[4]. Bei Patienten mit Morbus Meulengracht ist die Enzymaktivität der Bilirubin-UDP-Glucuronyltransferase auf etwa $30 \%$ der normalen Enzymaktivität reduziert[5, 6]. Der Morbus Meulengracht ist das häufigste familiäre Hyperbilirubinämiesyndrom. Ursache für die Hyperbilirubinämie ist eine Mutation im UGT1A1-Locus, der das Enzym Bilirubin-UDP-Glucuronyltransferase codiert. In der Promotorregion, der sogenannten TATA-Box findet sich eine Insertion von zwei zusätzlichen Basen. Die heterozygote Insertion in der Promotorregion kann mit minimal erhöhten Bilirubinspiegeln korrelieren, die homozygote Insertion führt zum Meulengracht-Syndrom[7, 8]. Die Krankheit wird autosomal-rezessiv vererbt. Man schätzt, dass 10-13 \% der kaukasischen Bevölkerung davon betroffen sind[9]. Der Morbus Meulengracht hat keinen eigentlichen Krankheitswert, es kann jedoch zu zahlreichen Beschwerden kommen. Es kann zu Ikterus mit einer Gelbfärbung der Haut, insbesondere der Skleren führen, was die Betroffenen vor Erhalt der Diagnose beunruhigen kann. Als auslösende Faktoren die bei Betroffenen zu einer Hyperbilirubinämie führen können werden Alkohol, Rauchen, Infektionen, Schlafmangel, Stress und Hungern angesehen.

Ein Aspekt der in den letzten Jahren immer mehr an Bedeutung gewonnen hat, ist dass eine verminderte Aktivität der Bilirubin-UDP-Glucuronyltransferase einen wichtigen Einfluss auf medikamentöse Therapien hat. Beispielsweise ist bei Patienten die an einem Morbus Meulengracht leiden unter einer Chemotherapie mit Irinotecan die Therapietoxizität deutlich erhöht[10]. Es gibt daher sogar Diskussionen ob bei Patienten bei denen eine Irinotecan Therapie geplant ist routinemässig eine UGT1A1 Genotypisierung durchgeführt werden sollte[11]. Auch unter Atazanavir Therapie, einem Proteasehemmer welcher in der HIV Therapie Verwendung findet, prädisponiert der Morbus Meulengracht für das Auftreten einer Hyperbilirubinämie[12].

Die Diagnose des Morbus Meulengracht kann, nach Ausschluss anderer Ursachen einer Hyperbilirubinämie, klinisch gestellt werden. Eine Genotypisierung des UGT1A1 Promoters kann die Diagnose bestätigen. Eine weitere Diagnosemöglichkeit besteht in Durchführung eines Nikotinsäure- oder Fastentests. Durch die Gabe von Nikotinsäure wird die Erythrozytenfragilität gesteigert, was zu einem erhöhten Anfall von unkonjugiertem Bilirubin führt. Beim Morbus Meulengracht kommt es dabei im Vergleich zum Gesunden zu einem stärkeren Anstieg des Bilirubins. Beim Fastentest kommt es bei Patienten mit Morbus Meulengracht unter Fastenbedingungen zu einem ansteigenden Blutwert für das indirekte Bilirubin. Jedoch finden diese beiden Tests kaum Verwendung, da die Diagnose wie bereits erwähnt auch klinisch gestellt werden kann.

\section{Studienaufbau}

Bei einer Kohorte des UniversitätsSpitals Zürich (USZ) wurden aufgrund von retrospektiv erhobenen Daten die klinischen Symptome und laborchemischen Veränderungen (Bilirubin) bei Patienten mit einem Meulengracht-Syndrom untersucht. Insgesamt wurden 46 Patienten aus der Datenbank des USZ untersucht, welche zwischen 2008 und 2010 einen positiven Nachweis einer homozygoten UGT1A1 Mutation aufwiesen. 
Es wurden Symptome wie Diarrhoe, Flatulenz, Müdigkeit, Kopfschmerzen, Magendruck und Darmkrämpfe welche mit einem Morbus Meulengracht in Verbindung gebracht werden können untersucht. Zusätzlich wurden verschiedene Trigger wie Alkohol, Rauchen, Infektionen, Schlafmangel, Stress und Hungern überprüft. Diese Symptome und Trigger wurden mit den jeweilig gemessenen Bilirubinwerten abgeglichen. Untersucht wurde das Gesamt-Bilirubin, da dieses in der Routinediagnostik Verwendung findet.

\section{Ergebnisse der Studie}

Von 2008 bis 2010 wurde am UniversitätsSpital in Zürich insgesamt 46-malig eine Genotypisierung des UGT1A1 Promoters veranlasst. Von diesen wurden 9 aufgrund eines heterozygoten UGT1A1 Mutation (4 Patienten) und dem Nachweis des Wildtyp UGT1A1 (5 Patienten) aus der Wertung genommen. Von den 37 untersuchten Patienten mit homozygoter UGT1A1 Promoter Mutation waren 30 männlich (81\%) und 7 weiblich (9\%), das Durchschnittsalter lag bei 40 Lebensjahren. Das durchschnittliche GesamtBilirubin lag bei 31.6 $\mu \mathrm{mol} / \mathrm{l}$. Bei einem Patienten war kein Bilirubin bestimmt worden, ein Patient wurde aus der Wertung genommen aufgrund eines B-Zelllymphoms mit Leberbefall.

Bei der Untersuchung von Triggern welche eine Hyperbilirubinämie im Rahmen des M. Meulengracht auslösen können konnte nur bei 2 Patienten (5.6\%) anhand der vorliegenden Dokumentationen Stress als Trigger beschrieben werden. Es fanden sich keine Hinweise auf Alkohol, Rauchen, Infektionen, Schlafmangel und Hungern als Trigger. Ein Patient mit Stress als Trigger verspürte als Symptome Übelkeit und Sklerenikterus, der andere Patient Blähungen, Oberbauchschmerzen, Übelkeit und Gliederschmerzen. Dies legt nahe, dass in der untersuchten Studienpopulation bis auf Stress keine Trigger der Beschwerden zu dokumentieren waren.

Um den Zusammenhang zwischen verschiedenen Symptomen und Morbus Meulengracht zu untersuchen wurde die Krankengeschichte nach dem Vorhandensein von unterschiedlichen Symptomen untersucht (vgl. Graphik 1). Insgesamt wurden Symptome bei 13 der 36 Untersuchten Patienten beobachtet (36.1\%), die Symptome von 4 Teilnehmern wurden aus der Wertung genommen, da ein anderes Leiden als ursächlich für die Beschwerden erachtet wurde. An Symptomen in der gesamten untersuchten Patientengruppe dominierten Oberbauchschmerzen bei 6 Teilnehmern $(16.7 \%)$ und ein Sklerenikterus bei 4 Teilnehmern (11.1\%). Übelkeit und nicht näher bezeichnete Abdominalschmerzen waren bei jeweils 2 Teilnehmern zu finden (5.6\%). Pruritus, Müdigkeit, Gliederschmerzen, Kopfschmerzen, Migräne, Muskelkrämpfe, Diarrhoe, Flatulenz und Stuhlunregelmässigkeiten konnten bei nur jeweils einem Studienteilnehmer (2.8\%) beobachtet werden (siehe Graphik 1). Bei Frauen traten Symptome bei 4 von 7 Teilnehmerinnen (57.1\%) verglichen mit und 12 von 29 Teilnehmern (41.4\%) bei den Männern deutlich häufiger auf. Jedoch sind die Unterschiede bei nur kleiner Zahl an untersuchten Frauen schwierig zu interpretieren. Um Assoziationen mit anderen Erkrankungen zu untersuchen wurde die persönliche Anamnese bezüglich verschiedener Erkrankungen untersucht. Gallensteine, eine Colitis ulcerosa, ein Ikterus intermittens juvenilis, eine Thalassämia minor und ein Reizdarm liessen sich jeweils nur bei einem Teilnehmer nachweisen (jeweils 2.8\%). Ein Teilnehmer litt an einer chronischen Hepatitis C mit Leberfibrose. Daher konnte keine direkte Assoziation zu einem dieser Krankheitsbilder gefunden werden. 
Bei den Teilnehmern mit Symptomen lag das Gesamt-Bilirubin bei 33.7 $\mu \mathrm{mol} / \mathrm{l}$ (15-75 $\mu \mathrm{mol} / \mathrm{l}) \mathrm{im}$ Vergleich zu 31.8 $\mu \mathrm{mol} / \mathrm{l}$ (8.2-59.6 $\mu \mathrm{mol} / \mathrm{l})$ bei Teilnehmern ohne Symptome. Die Verteilung des Gesamt-Bilirubins war bei den Studienteilnehmern mit Symptomen in einem höheren Bereich angeordnet, jedoch nicht signifikant $(p=0.2117)$ (vgl. Graphik 2). Wir schliessen daraus, dass anhand des Gesamt-Bilirubinwertes kein Rückschluss auf das Vorhandensein von Symptomen gemacht werden kann.

\section{Diskussion}

Insgesamt zeigt sich, dass bei nur 46 Genotypisierungen des UGT1A1 Promoters in einem Zeitraum von 3 Jahren (2008-2010) am UniversitätsSpital, diese Bestimmung nur selten durchgeführt wird, und das obwohl von einer Prävalenz des Morbus Meulengracht von 4-5\% ausgegangen wird. Bei 37 von 46 Genotypisierungen des UGT1A1 Promoters aufgrund einer Abklärung einer isolierten Hyperbilirubinämie konnte eine homozygote Mutation beschrieben werden. Daher zeigt sich eine gute Übereinstimmung zwischen klinischem Verdacht auf einen Morbus Meulengracht und dem Nachweis einer homozygoten UGT1A1 Mutation.

Die Genotypisierung des UGT1A1 Promoters ist daher ein wichtiger Schritt in der Abklärung einer isolierten Hyperbilirubinämie unbekannter Ursache. Sie sollte dann durchgeführt werden wenn Unklarheiten bezügliche der Ätiologie einer isolierten Hyperbilirubinämie bestehen, nachdem andere Ursachen wie Hämolyse, medikamentöstoxische Ursachen und virale Hepatitiden ausgeschlossen worden sind. Die molekulare Diagnose kann hiermit korrekt gestellt werden, ohne dass invasive Abklärungen, wie Leberbiopsien, benötigt werden.

Aufgrund des Einflusses einer verminderten Aktivität der Bilirubin-UDPGlucuronyltransferase auf bestimmte medikamentöse Therapien, sollte beispielsweise bei klinischem Verdacht auf einen Morbus Meulengracht vor Beginn einer Chemotherapie mit Irinotecan eine UGT1A1 Genotypisierung durchgeführt werden. 


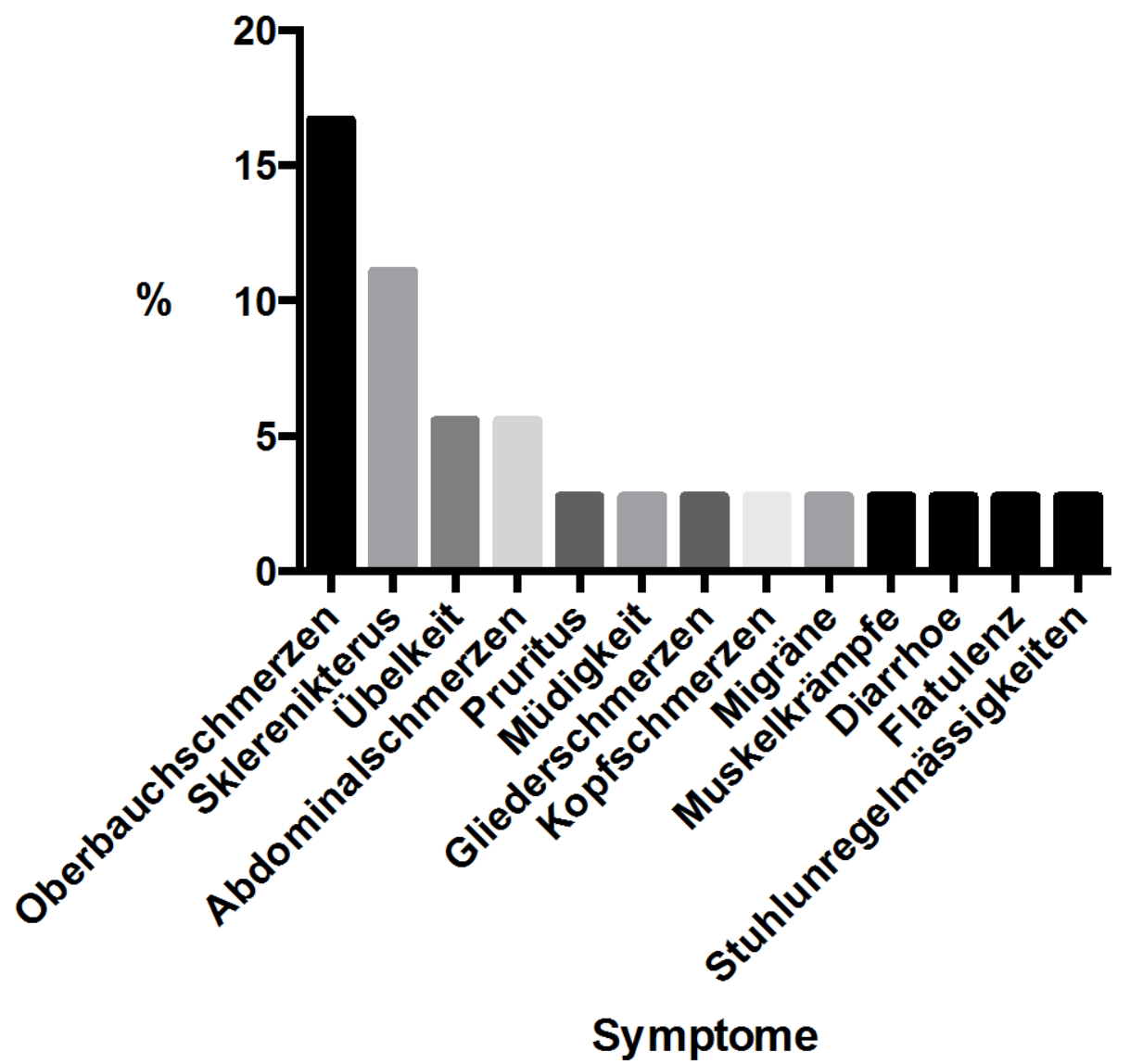

Graphik 1: Häufigkeit des Auftretens von Symptomen in Prozent in der untersuchten Studienpopulation

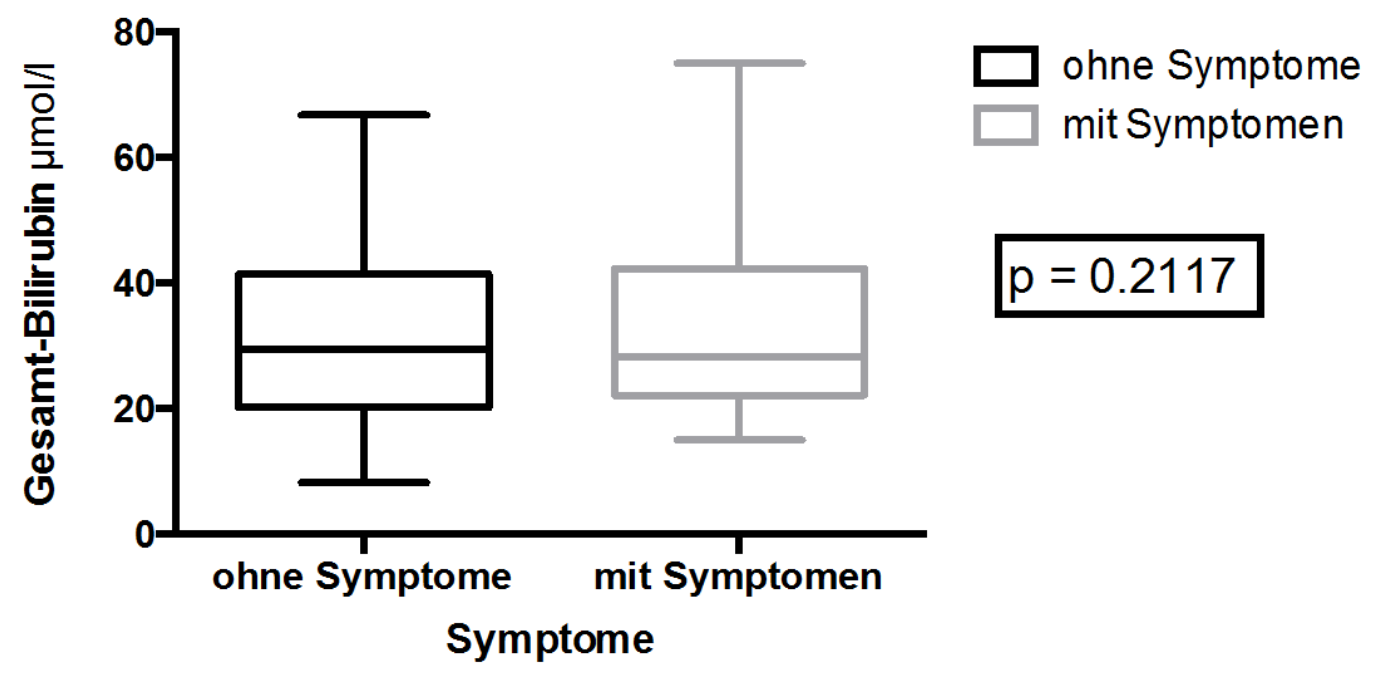

Graphik 2: Darstellung der Verteilung des Gesamt-Bilirubins bei Studienteilnehmern mit und ohne Symptome. 


\section{Fallbericht zu Morbus Meulengracht}

\section{Anamnese des Patienten}

Wir berichten über einen 40 jährigen Patienten. Der Patient arbeitet als Berater und ist daher häufig auf Reisen. Der Patient stellte sich im Oktober 2009 in der gastroenterologischen Sprechstunde aufgrund von rezidivierenden Oberbauchbeschwerden vor. Diese äusserten sich als intermittierende Druckdolenz im rechten Oberbauch, verbunden mit leichter Übelkeit, insbesondere wenn der Patient im Rahmen beruflicher Reisetätigkeit unregelmässige Mahlzeiten zu sich nimmt. Zusätzlich waren der Ehefrau des Patienten, sowie auch den Arbeitskollegen, eine intermittieremde leichte Gelbfärbung der Skleren aufgefallen. Das Gewicht des Patienten war stabil, Nachtschweiss und Stuhlunregelmässigkeiten wurden verneint. Seine persönliche Anamnese umfasste eine stattgehabte Appendektomie in der Kindheit, die Familienanamnese war unauffällig. Im Rahmen der beruflichen Reisetätigkeit wurden in den letzten 5 Jahren Deutschland, Österreich und Frankreich bereist. Die einzigen Reisen in ferne Länder umfassten die USA (New York) vor 7 und Grossbritannien (London) vor 6 Jahren. An Medikamenten wurde vom Patienten nur Paracetamol in Reserve (ca. 1-2 Tabletten zu 1g pro Woche) eingenommen. Der Alkoholkonsum beschränkt sich auf wenig Wein zum Essen, ca. 2 Gläser Weiss- oder Rotwein pro Woche.

Im klinischen Status ergab sich bis auf einen diskreten Sklerenikterus ein unauffälliger Befund. Laborchemisch zeigten sich ein normales Blutbild, Kreatinin, CRP LDH und Transaminasen (AST/ALT) bei leichter indirekter Hyperbilirubinämie.

\section{Weg zur Diagnose/Kommentar}

Es bestand, bis auf einen leichten Sklerenikterus, ein unauffälliger klinischer Untersuchungsbefund. Laborchemisch ergab sich kein Hinweis auf ein entzündliches Geschehen. Bei fehlender B-Symptomatik auch kein Hinweis auf ein neoplastisches Geschehen.

\section{Befunde des Patienten}

\section{Status}

40-jähriger Patient in gutem Allgemeinzustand, Blutdruck 128/78mmHg, Temperatur $36.8^{\circ} \mathrm{C}$. Normale Herztöne ohne Nebengeräusche. Normales Atemgeräusch ohne Nebengeräusche. Abdomen weich mit normalen Darmgeräuschen, keine Resistenzen, keine Druckdolenz, Murphy-Zeichen negativ, digital rektale Untersuchung unauffällig.

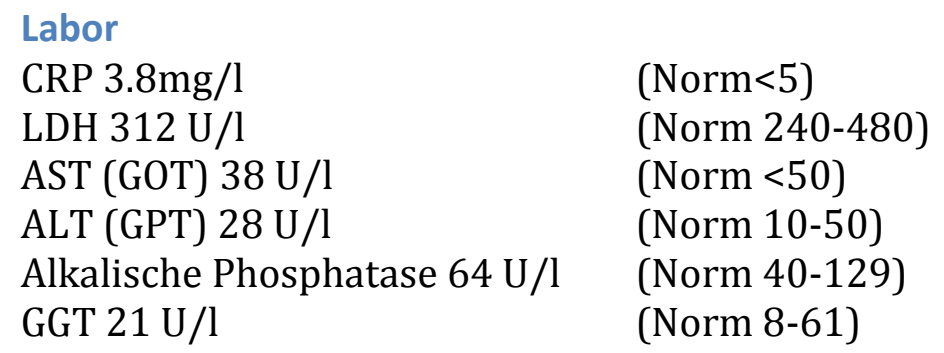


Pankreasamylase $31 \mathrm{U} / \mathrm{l}$

Bilirubin, total $30 \mu \mathrm{mol} / \mathbf{l}$

Bilirubin, direkt $2.1 \mu \mathrm{mol} / \mathrm{l}$

Hämoglogin 136g/dl

Leukozyten $3.94 \mathrm{G} / \mathrm{l}$

(Nierenretentionsparam
(Norm 13-53)

(Norm $<21$ )

(Norm $<3.4$ )

(Norm 134-170)

(Norm 3.0-9.6)

\section{Weg zur Diagnose/Kommentar}

Laborchemisch zeigen sich keine erhöhten Entzündungsparameter, somit kein Hinweis auf ein florides infektiologisches Geschehen. Es zeigt sich isoliert eine indirekte Bilirubinerhöhung bei normaler GGT und Alkalischer Phosphatase. Bei normalem direktem Bilirubin ergibt sich kein Hinweis für eine Cholestase. Eine relevante Reisenanamnese lag nicht vor. Es ergab sich zusätzlich kein Hinweis auf eine Hämolyse bei normwertiger LDH und unauffälligem Blutbild. Differentialdiagnostisch muss bei dieser Konstellation, trotz normwertigen Transaminasen, eine virale Hepatitis ausgeschlossen werden, auch wenn diese typischerweise mit einer direkten Bilirubinerhöhung assoziiert ist. Zusätzlich sollte eine erweiterte Medikamentenanamnese erfolgen, um eine medikamentös-toxische Ursache auszuschliessen. Des Weiteren muss an eine hereditäre Ursache der indirekten Hyperbilirubinämie gedacht werden. Ein Crigler-Najjar Syndrom ist bei nur leichter Beschwerdesymptomatik und nur leicht erhöhtem Bilirubin unwahrscheinlich.

\section{Weiterer Verlauf}

Bei dem Patienten wurde eine Hepatitis-Serologie durchgeführt, bei der eine Hepatitis A, $B$ und $C$ ausgeschlossen werden konnten. In der erweiterten Medikamentenanamnese ergab sich ein unauffälliger Befund, ohne Einnahme von beispielsweise VitaminPräparaten, Ajurveda-Tees oder chinesischen Kräutern. Zur weiteren Evaluation eines möglichen Morbus Meulengracht wurde eine Genotypisierung des UGT1A1 Promoters veranlasst welche eine Homozygotie für UGT1A1 Promoter (TA) 7- Mutation nachweisen konnte. Zusammenfassend ist daher von einem Morbus Meulengracht bei dem Patienten auszugehen. Der Patient wurde über die Gutartigkeit der Erkrankung aufgeklärt. Nachdem der Patient begann auf eine regelmässige Nahrungsaufnahme ohne längere Fastenzeiten zu achten waren die rechtsseitigen Oberbauchbeschwerden komplett rückläufig.

\section{Fragen zu Morbus Meulengracht}

1) Welche Testmethoden bestehen bei klinischem Verdacht auf einen Morbus Meulengracht? (Mehrfachauswahl möglich)
a. Nikotinsäure-Test
b. Fasten-Test
c. Glucosetoleranz-Test
d. Genetische Bestimmung des UGT1A1 Gens

Auflösung: richtig sind: $a, b$ und $d$ 
Beim Nikotinsäure- und Fastentest kommt es zu ansteigendem Blutwert für das indirekte Bilirubin. Die Tests haben für die Diagnose keine grosse Bedeutung, da die Diagnose auch ohne diese Tests bestimmt werden kann. Des Weiteren besteht die Möglichkeit durch eine genetische Bestimmung eine Mutation im UGT1A1 Genlocus festzustellen. Ursache ist eine Mutation im UGT1A1Locus, der das Enzym Bilirubin-UDP-Glucuronyltransferase codiert. In der Promotorregion, der sogenannten TATA-Box des Exons 1A findet sich eine Insertion von zwei zusätzlichen Basen. Die heterozygote Insertion in der Promotorregion kann mit minimal erhöhten Bilirubinspiegeln korrelieren, die homozygote Insertion führt zum Meulengracht-Syndrom.

2) Nach welchem Muster wird der Morbus Meulengracht vererbt?

a. Autosomal-dominant

b. Autosomal-rezessiv

c. X-chromosomal

d. Y-chromosomal

Auflösung: Die Krankheit wird autosomal-rezessiv vererbt. Man schätzt, dass 4 - 5 \% der Bevölkerung davon betroffen sind.

3) Auf welchen Wert ist die Enzymaktivität beim Morbus Meulengracht reduziert?
a. Ca. $10 \%$
b. Ca. $30 \%$
c. Ca. $60 \%$
d. Ca. $80 \%$

Auflösung: Bei Patienten mit Morbus Meulengracht ist die Enzymaktivität der Bilirubin-UDP-Glucuronyltransferase auf etwa $30 \%$ der normalen Enzymaktivität reduziert.

4) Wie unterscheidet sich das Crigler-Najjar-Syndrom vom Morbus Meulengracht

a. Crigler-Najjar-Syndrom und Morbus Meulengracht sind Synonyme für die gleiche Erkrankung

b. Das Crigler-Najjar-Syndrom ist im Gegensatz zum Morbus Meulengracht gekennzeichnet durch eine direkte Hyperbilirubinämie

c. Der Enzymdefekt beim Crigler-Najjar-Syndrom ist deutlich schwerer ausgeprägt als beim Morbus Meulengracht

d. Das Crigler-Najjar-Syndrom manifestiert sich später im Leben als Morbus Meulengracht.

Auflösung: Der Enzymdefekt beim Crigler-Najjar-Syndrom ist deutlich schwerer ausgeprägt als beim Morbus Meulengracht. Beim Crigler-Najjar-Syndrom unterscheidet man Typ I und II. Der Typ I wird autosomal-rezessiv vererbt und ist charakterisiert durch ein komplettes Fehlen des Enzyms Bilirubin-UDPGlucuronosyltransferase. Der Typ II wird autosomal-rezessiv vererbt, dort 
besteht noch eine geringe Restaktivität des Enzyms Bilirubin-UDPGlucuronyltransferase.

5) Welche weitere Diagnostik ist indiziert sobald die Diagnose eine Morbus Meulengracht gestellt werden konnte
a. Leberbiopsie
b. Genetische Abklärung der Familienmitglieder
c. Jährliche Sonographie der Leber
d. keine weitere Diagnostik indiziert

Auflösung: Der Morbus Meulengracht hat keinen eigentlichen Krankheitswert, eine weiterführende Diagnostik ist nicht erforderlich.

\section{Literatur}

1. $\quad$ Manfred Dietel, N.S., Martin Zeitz, Harrisons Innere Medizin 17. Auflage, 2009.

2. Sampietro, M. and A. Iolascon, Molecular pathology of Crigler-Najjar type I and II and Gilbert's syndromes. Haematologica, 1999. 84(2): p. 150-7.

3. Gilbert A, L.P., La cholémie simple familiale. Semaine Médicale, 1906. 21: p. 241-5.

4. Strassburg, C.P., S. Kalthoff, and U. Ehmer, Variability and function of family 1 uridine-5'-diphosphate glucuronosyltransferases (UGT1A). Critical reviews in clinical laboratory sciences, 2008. 45(6): p. 485-530.

5. Arias, I.M. and I.M. London, Bilirubin glucuronide formation in vitro; demonstration of a defect in Gilbert's disease. Science, 1957. 126(3273): p. 563-4.

6. Black, M. and B.H. Billing, Hepatic bilirubin udp-glucuronyl transferase activity in liver disease and gilbert's syndrome. The New England journal of medicine, 1969. 280(23): p. 1266-71.

7. Rodrigues, C., et al., Impact of UGT1A1 gene variants on total bilirubin levels in Gilbert syndrome patients and in healthy subjects. Blood cells, molecules \& diseases, 2012. 48(3): p. 166-72.

8. Kadakol, A., et al., Genetic lesions of bilirubin uridine-diphosphoglucuronate glucuronosyltransferase (UGT1A1) causing Crigler-Najjar and Gilbert syndromes: correlation of genotype to phenotype. Human mutation, 2000. 16(4): p. 297-306.

9. Borlak, J., et al., Molecular diagnosis of a familial nonhemolytic hyperbilirubinemia (Gilbert's syndrome) in healthy subjects. Hepatology, 2000. 32(4 Pt 1): p. 792-5.

10. Strassburg, C.P., Pharmacogenetics of Gilbert's syndrome. Pharmacogenomics, 2008. 9(6): p. 703-15.

11. Palomaki, G.E., et al., Can UGT1A1 genotyping reduce morbidity and mortality in patients with metastatic colorectal cancer treated with irinotecan? An evidencebased review. Genetics in medicine : official journal of the American College of Medical Genetics, 2009. 11(1): p. 21-34.

12. Lankisch, T.O., et al., Gilbert's disease and atazanavir: from phenotype to UDPglucuronosyltransferase haplotype. Hepatology, 2006. 44(5): p. 1324-32. 